\title{
Perioperative Magnesium for Postoperative Analgesia: An Umbrella Review of Systematic Reviews and Updated Meta-Analysis of Randomized Controlled Trials
}

\author{
Geun Joo Choi ${ }^{1}$, Young Il Kim ${ }^{1}$, Young Hyun $\mathrm{Koo}^{1}$, Hyoung-Chul Oh ${ }^{2}$ and Hyun Kang ${ }^{1, *(D)}$ \\ 1 Department of Anesthesiology and Pain Medicine, Chung-Ang University College of Medicine, \\ Seoul 06911, Korea; pistis23@naver.com (G.J.C.); turtlebay7@naver.com (Y.I.K.); \\ yhkoo28@caumc.or.kr (Y.H.K.) \\ 2 Division of Gastroenterology, Department of Internal Medicine, Chung-Ang University College of Medicine, \\ Seoul 06911, Korea; ohcgi@cau.ac.kr \\ * Correspondence: roman00@naver.com; Tel.: +82-2-6299-2586
}

\section{check for} updates

Citation: Choi, G.J.; Kim, Y.I.; Koo, Y.H.; Oh, H.-C.; Kang, H.

Perioperative Magnesium for

Postoperative Analgesia: An

Umbrella Review of Systematic

Reviews and Updated Meta-Analysis of Randomized Controlled Trials. J. Pers. Med. 2021, 11, 1273. https:// doi.org/10.3390/jpm11121273

Academic Editors: Young-Kug Kim and Kitae Jung

Received: 9 October 2021

Accepted: 19 November 2021

Published: 2 December 2021

Publisher's Note: MDPI stays neutral with regard to jurisdictional claims in published maps and institutional affiliations.

Copyright: (c) 2021 by the authors. Licensee MDPI, Basel, Switzerland. This article is an open access article distributed under the terms and conditions of the Creative Commons Attribution (CC BY) license (https:/ / creativecommons.org/licenses/by/ $4.0 /)$.

\begin{abstract}
The purpose of this study was to summarize and evaluate evidence on the effectiveness of perioperative magnesium as an adjuvant for postoperative analgesia. We conducted an umbrella review of the evidence across systematic reviews and meta-analyses of randomized controlled trials (RCTs) on the effect of perioperative magnesium on pain after surgical procedures. Two independent investigators retrieved pain-related outcomes and assessed the methodological quality of the evidence of included studies using the A MeaSurement Tool to Assess systematic Reviews (AMSTAR) tool, and the Grading of Recommendations, Assessment, Development, and Evaluation (GRADE) system. In addition, an updated meta-analysis of postoperative pain-related outcomes with a trial sequential analysis (TSA) was conducted. Of the 773 articles initially identified, 17 systematic reviews and meta-analyses of 258 RCTs were included in the current umbrella review. Based on the AMSTAR tool, the overall confidence of the included systematic reviews was deemed critically low to low. Pain score, analgesic consumption, time to first analgesic request, and incidence of analgesic request were examined as pain-related outcomes. According to the GRADE system, the overall quality of evidence ranged from very low to moderate. While the updated meta-analysis showed the beneficial effect of perioperative magnesium on postoperative analgesia, and TSA appeared to suggest sufficient existing evidence, the heterogeneity was substantial for every outcome. Although the majority of included systematic reviews and updated meta-analysis showed a significant improvement in outcomes related to pain after surgery when magnesium was administered during the perioperative period, the evidence reveals a limited confidence in the beneficial effect of perioperative magnesium on postoperative pain.
\end{abstract}

Keywords: analgesia; magnesium; meta-analysis; pain; postoperative; systematic review; umbrella review

\section{Introduction}

Postoperative pain can be linked to various pathophysiological pathways, including neuropathic and inflammatory pathways [1]. These various factors can be considered when developing a pain-management strategy following surgery. In this regard, there is a growing interest in the use of multimodal analgesia as an important component of ideal methods for postoperative pain management [2].

Magnesium has been shown to have analgesic benefits when used as an adjuvant in surgical patients and can therefore contribute to being part of a balanced analgesia strategy $[3,4]$. The regulation of calcium influx into the cell and antagonism of $N$-methyl-Daspartate (NMDA) receptors in the central nervous system are assumed to be responsible for magnesium's analgesic activity; however, the exact mechanism remains uncertain $[5,6]$. 
Numerous investigations have yielded contradictory findings since the first study on magnesium sulfate as an analgesic adjuvant in 1996 [4].

A number of systematic reviews and meta-analyses have been published that investigated the effect of magnesium administration during the perioperative period on postoperative pain. There has been no study to our knowledge that summarizes the evidence from these systematic reviews and meta-analyses. As a result, we re-evaluated the evidence in a systematic and comprehensive manner to offer an overview of the effect of perioperative magnesium administration on postoperative pain. We performed a qualitative umbrella review to analyze the findings of systematic reviews and meta-analyses that evaluated the efficacy of perioperative magnesium on postoperative pain as well as evidence of potential limits and consistency of results. We additionally conducted a quantitative meta-analysis including the latest data to update the existing evidence, which was also evaluated by trial sequential analysis.

\section{Materials and Methods}

An umbrella review summarizes previously published systematic reviews and metaanalyses to determine whether authors addressing comparable review issues have independently reported similar findings and reached comparable conclusions [7]. We conducted a literature review focusing on the effectiveness of perioperative magnesium as an adjuvant for postoperative pain. We also performed an updated meta-analysis including data from the last study. This study was prospectively registered with the PROSPERO database of systematic reviews (CRD42021265991) and conducted according to the Preferred Reporting Items for Systematic Reviews and Meta-Analyses (PRISMA) guidelines [8].

\subsection{Search Strategy}

We systematically searched MEDLINE, EMBASE, Cochrane Database of Systematic Reviews, and Scopus from inception to the end of March 2021 and updated on 1 July. A list of references from eligible systematic reviews and related reviews were scanned for additional citations. There were no language restrictions.

\subsection{Eligibility Criteria}

We included systematic reviews and meta-analyses reporting the effects of perioperative administration of magnesium as an adjuvant for postoperative pain. Two independent investigators (G.J.C. and Y.I.K.) assessed the following eligibility criteria: (1) Participants: adults and pediatric patients underwent surgery under general or regional anesthesia; (2) Intervention: magnesium administration as an adjuvant for postoperative pain management; (3) Comparison: placebo, no treatment, or no magnesium administration as control group; (4) Outcomes (summary measures for pain-related outcomes during postoperative period): pain score, analgesic consumption, time to analgesic request, and incidence of rescue analgesic; (5) Study design: systematic reviews and/or meta-analyses of randomized controlled trials (RCTs). In case of disagreement, all issues were discussed with a third investigator (H.K.). Narrative and other types of non-systematic reviews (e.g., critical reviews, overviews, state-of-the-art reviews), clinical practice guidelines, evidence summaries, critically appraised topics, clinical paths, consumer information sheets, best practice information sheets, technical reports, and other evidence-based pieces were excluded.

\subsection{Data Extraction}

Two investigators (G.J.C. and Y.I.K.) extracted the following from each eligible study: (1) first author, (2) year of publication, (3) name of journal, (4) number of included RCTs, (5) number of participants in each trial arm, (6) information on intervention and comparison, (7) surgical procedure, (8) information on outcomes reported as primary or secondary, and (9) type of effect size used in the meta-analysis (effect size with $95 \%$ confidence intervals $(95 \%$ Cis)). We finally extracted the information required by the Assessment of Multiple Systematic Reviews (AMSTAR) tool [9]. 


\subsection{Assessment of the Confidence and Quality of Evidence}

The overall confidence of the included systematic reviews was determined using AMSTAR (A MeaSurement Tool to Assess systematic Reviews) version 2.0 [10]. This revised tool simplifies response categories and contains 16 items in all, which provide a more comprehensive appraisal compared with the original AMSTAR [9]. The quality of evidence for each pooled outcome from the included systematic reviews was assessed using GRADE (Grading of Recommendations Assessment, Development, and Evaluation) [11]. In this approach, the quality of evidence was categorized as high, moderate, low, or very low based on limitations in risk of bias, precision, consistency, directness, and publication bias.

AMSTAR evaluation and GRADE classification were independently performed by two investigators (G.J.C. and H.C.O.). Any discrepancy was resolved via discussion, and all discrepancies that could not be resolved through discussion were arbitrated by a third investigator (H.K.).

\subsection{Updated Meta-Analysis}

A study search was performed based on RCTs included in the systematic reviews of umbrella reviews and newly published RCTs since January 2020. RCTs were included in this updated meta-analysis if they compared perioperative magnesium administration with the control group in patients undergoing surgical procedures under anesthesia. There were no language restrictions, and postoperative pain-related outcomes were extracted. Two investigators (G.J.C. and Y.H.K.) independently performed the study search, study selection, and data extraction. In case of disagreement, all issues were discussed with a third investigator (H.K.).

\subsection{Statistical Analysis}

The pooled risk ratio (RR) and 95\% confidence intervals (CIs) for incidence of rescue analgesic and standardized mean difference (SMD) and 95\% CIs for pain score, analgesic consumption, and time to analgesic request were calculated, respectively. In terms of pain scores, visual analogue scale and numerical rating scale in adult patients and Children's Hospital of Eastern Ontario Pain Scale in pediatric patients were utilized, respectively, which were standardized in outcome synthesis of updated meta-analysis. We used the chi-square test for homogeneity and the $\mathrm{I}^{2}$ test for heterogeneity. We regarded a level of $10 \%$ significance $(p<0.100)$ in the $\chi^{2}$ statistic or an $\mathrm{I}^{2}$ greater than $50 \%$ as considerable heterogeneity. For both categorical and continuous data, we used the DerSimnian-Laird random-effect model. Otherwise, we applied the fixed-effect model. As pain score data were measured at multiple time points, we adopted two strategies to select and analyze the data. First, we selected the data of the nearest time point from the specified time point: $0 \mathrm{~h}$ postoperatively for the PACU phase, $4 \mathrm{~h}$ postoperatively for the early phase, and $24 \mathrm{~h}$ postoperatively for the late phase. Second, as data measured at multiple time points were dependent on each other, and multiple comparisons at each time point would increase the possibility of type I error, we combined outcomes from multiple time points within specified periods (namely $0-1 \mathrm{~h}$ for PACU and $0-4 \mathrm{~h}$ for early phase) and performed the analysis using the pooled combined outcomes. Publication bias was evaluated using funnel plots and Egger's linear regression test. If the funnel plot was asymmetrical, or the $p$-value was $<0.100$ by Egger's test, we considered the presence of a publication bias and performed trim-and-fill analysis.

As traditional meta-analysis runs the risk of random errors due to sparse data, we additionally performed a trial sequential analysis (TSA). TSA is a methodology that includes a required information size (RIS) calculation for a meta-analysis with a threshold for statistical significance, which controls the risk of potential false-positive and false-negative findings of meta-analyses and provides information on whether the results of our study were conclusive. We used a fixed or random effects model to construct a cumulative Z-curve. TSA was performed to maintain an overall $5 \%$ risk of type I error. Meta-analysis 
was conducted using Comprehensive Meta-Analysis version 2.0 (Englewood, NJ, USA, 2008) and TSA 0.9.5.10 $\beta$ software (Copenhagen Trial Unit, Copenhagen, Denmark).

\section{Results}

\subsection{Description of Included Systematic Reviews}

Of the 773 articles initially identified, 21 full-text articles were assessed for eligibility. Four studies were excluded because there was no report regarding pain-related outcome during postoperative period [12-15]. Seventeen systematic reviews, including 258 individual RCT estimates, were finally selected for this umbrella review. The study selection process and reasons for exclusion are showed in Figure 1. Participants were pregnant women in two studies [16,17], pediatric patients in three [18-20], and adult patients in the others. Magnesium as an adjuvant was administered pre- and/or intraoperatively in the intervention group, while placebo or nothing in comparison with magnesium was administered in the control group. Sixteen included studies were systematic reviews and meta-analyses of RCTs, while one study was a systematic review of RCTs [21]. Routes of magnesium administration were intravenous in seven studies [21-27], intrathecal and/or epidural in five $[16,17,28-30]$, and intra-articular in two [31,32]. There were several routes, including intravenous, intrathecal, epidural, and local approaches, in three studies $[17,18,20]$. The types of surgery were cesarean section in two studies $[16,17]$, tonsillectomy in two [18,20], arthroscopic surgery in two [31,32], and laparoscopic cholecystectomy in one [23]. The remaining patients underwent multiple surgical procedures. The study characteristics of the adult and pediatric patients are summarized in Table 1.

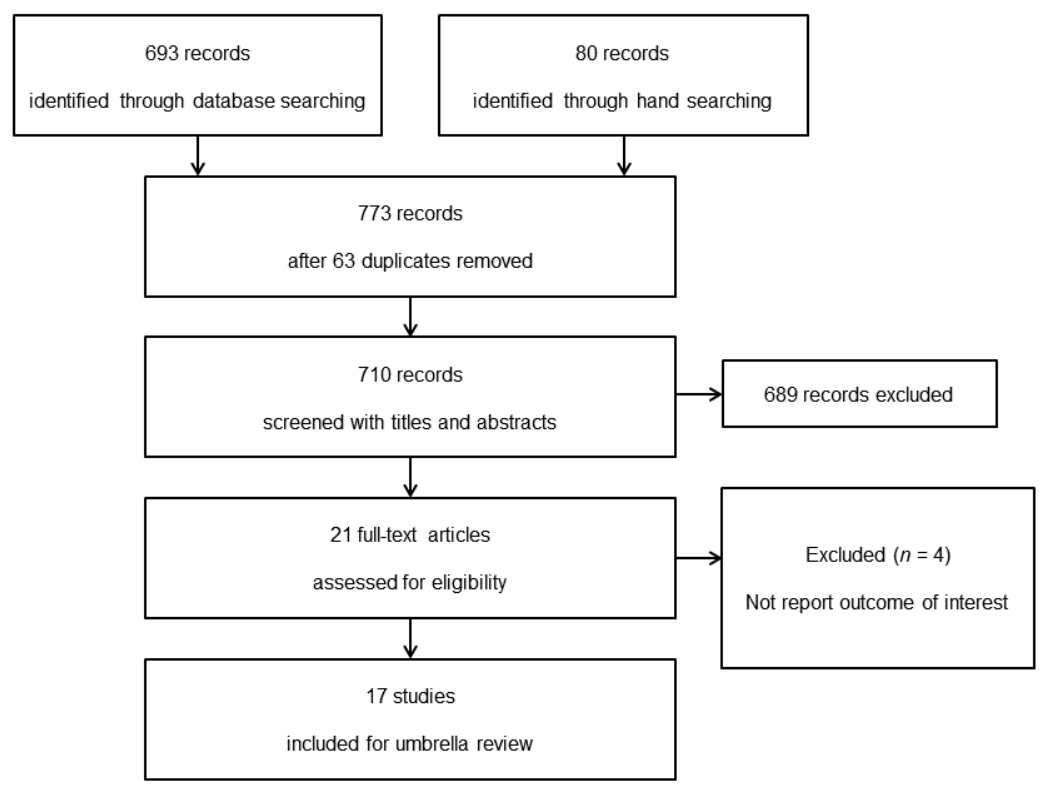

Figure 1. PRISMA flow chart of umbrella review.

\subsection{Summary of the Evidences}

Pain-related outcomes were pain score, analgesic consumption, time to first analgesic request, and number of patients requiring rescue analgesics after surgery. Ten studies in adult patients $[16,17,22-27,31,32]$ and two studies in pediatric patients $[18,20]$ conducted a meta-analysis regarding pain score after surgery. The majority of studies described using magnesium as part of a multimodal analgesic regimen to reduce postoperative pain intensity within $24 \mathrm{~h}$ of surgery. The pain score in adult patients showed a significant decrease in the magnesium group both at rest and during movement in a large body of meta-analyses, but heterogeneity was substantial. In contrast, the pain score in pediatric patients did not show any significant difference between the two groups. Summarized evidence on pain scores in adult and pediatric patients is shown in Table 2. 
Table 1. Study characteristics in adult and pediatric patients.

\begin{tabular}{|c|c|c|c|c|c|c|c|}
\hline $\begin{array}{l}\text { Patients } \\
\text { Group }\end{array}$ & First Author (Year) & Search Period & $\begin{array}{c}\text { Type of } \\
\text { Anesthesia }\end{array}$ & $\begin{array}{c}\text { Route of } \\
\text { Magnesium } \\
\text { Administration }\end{array}$ & $\begin{array}{c}\text { Number of } \\
\text { Included RCT }\end{array}$ & $\begin{array}{c}\text { Number of } \\
\text { Participant (Mag- } \\
\text { nesium/Control) }\end{array}$ & Type of Surgery \\
\hline \multirow{13}{*}{$\begin{array}{c}\text { Adult } \\
\text { patients }\end{array}$} & Shi (2021) & October, 2020 & GA RA & IA & 11 & $677(343 / 334)$ & $\begin{array}{l}\text { Arthroscopic knee } \\
\text { surgery }\end{array}$ \\
\hline & Мa (2021) & February, 2020 & GA RA & IV, IT, ED, local & 8 & $880(440 / 440)$ & Cesarean section \\
\hline & Wang (2020) & March, 2020 & RA & IT & 10 & $720(360 / 360)$ & Surgery procedure \\
\hline & Li (2020) & October, 2019 & RA GA + RA & ED & 11 & $724(362 / 362)$ & Surgical procedure \\
\hline & $\mathrm{Ng}(2020)$ & January, 2019 & GA RA & IV & 51 & 3311 & Non-cardiac surgery \\
\hline & Chen (2018) & June, 2018 & GA & IV & 4 & $263(131 / 132)$ & $\begin{array}{c}\text { Laparoscopic } \\
\text { cholecystectomy }\end{array}$ \\
\hline & Wang (2017) & November, 2016 & RA & IT, ED & 9 & 827 & Cesarean section \\
\hline & Zeng (2016) & January, 2016 & GA & IA & 8 & $513(242 / 271)$ & Arthroscopic surgery \\
\hline & Guo (2015) & September, 2014 & GA RA & IV & 27 & 1504 & Surgical procedure \\
\hline & De Oliveira (2013) & June, 2012 & GA & IV & 20 & $1257(639 / 618)$ & Surgical procedure \\
\hline & Albrecht (2013) & January, 2012 & GA RA & IV & 25 & $1461(731 / 730)$ & Surgical procedure \\
\hline & $\begin{array}{c}\text { Pascual-Ramirez } \\
\text { (2013) }\end{array}$ & December, 2011 & RA & IT & 12 & $817(412 / 405)$ & $\begin{array}{l}\text { Below-umbilicus } \\
\text { procedure }\end{array}$ \\
\hline & Murphy (2013) & July, 2011 & GA RA & IV & 22 & $1177(599 / 578)$ & Surgery procedure \\
\hline \multirow{3}{*}{$\begin{array}{l}\text { Pediatric } \\
\text { patients }\end{array}$} & Kawakami (2018) & November, 2017 & RA & ED & 6 & $371(179 / 192)$ & Surgical procedure \\
\hline & Xie (2017) & June, 2016 & GA & IV, local & 10 & $665(333 / 332)$ & Tonsillectomy \\
\hline & Cho (2017) & January, 2017 & GA & IV, local & 10 & $655(328 / 327)$ & Tonsillectomy \\
\hline
\end{tabular}

RCT, randomized controlled trial; GA, general anesthesia; RA, regional anesthesia; IA, intra-articular; IV, intravenous; IT, intrathecal; ED, epidural.

Table 2. Summary of evidence: pain score in adult patients.

\begin{tabular}{|c|c|c|c|c|c|c|}
\hline First Author, Year & Outcome & Study N & $\begin{array}{l}\text { Participant N } \\
\text { (Mg/Control) }\end{array}$ & MD, SMD, ES (95\% CI) & Heterogeneity & $\begin{array}{c}\text { Quality of } \\
\text { Evidence (GRADE) }\end{array}$ \\
\hline \multirow{10}{*}{ Shi 2021} & At rest & & & & & \\
\hline & $2 \mathrm{~h}$ & 8 & $423(212 / 211)$ & $\mathrm{MD}-0.74(-0.84,-0.64)$ & $\mathrm{I}^{2}=0 \%, p=0.51$ & Low \\
\hline & $4 \mathrm{~h}$ & 6 & $303(152 / 151)$ & $\mathrm{MD}-0.24(-0.37,-0.11)$ & $\mathrm{I}^{2}=0 \%, p=0.51$ & Moderate \\
\hline & $12 \mathrm{~h}$ & 6 & $304(152 / 152)$ & $\mathrm{MD}-0.53(-0.64,-0.41)$ & $\mathrm{I}^{2}=0 \%, p=0.51$ & High \\
\hline & $24 \mathrm{~h}$ & 7 & $372(186 / 186)$ & $\mathrm{MD}-0.33(-0.42,-0.24)$ & $\mathrm{I}^{2}=0 \%, p=0.51$ & High \\
\hline & At movement & & & & & \\
\hline & $2 \mathrm{~h}$ & 7 & $279(140 / 139)$ & $\mathrm{MD}-0.46(-0.64,-0.27)$ & $\mathrm{I}^{2}=0 \%, p=0.51$ & High \\
\hline & $4 \mathrm{~h}$ & 6 & $299(150 / 149)$ & $\mathrm{MD}-0.85(-1.40,-0.30)$ & $\mathrm{I}^{2}=0 \%, p=0.51$ & Moderate \\
\hline & $12 \mathrm{~h}$ & 6 & $299(150 / 149)$ & $\mathrm{MD}-0.83(-1.17,-0.48)$ & $\mathrm{I}^{2}=0 \%, p=0.51$ & Moderate \\
\hline & $24 \mathrm{~h}$ & 7 & $339(170 / 169)$ & $\mathrm{MD}-0.58(-0.79,-0.36)$ & $\mathrm{I}^{2}=0 \%, p=0.51$ & High \\
\hline \multirow{2}{*}{ Ma 2021} & Highest VAS & 8 & $880(440 / 440)$ & $\mathrm{MD}-0.74(-1.03,-0.46)$ & $\mathrm{I}^{2}=91.7 \%, p<0.001$ & \multirow[b]{2}{*}{ Low } \\
\hline & Last VAS & 8 & $880(440 / 440)$ & $\mathrm{MD}-0.47(-0.71,-0.23)$ & $\mathrm{I}^{2}=95.0 \%, p<0.001$ & \\
\hline Ng 2020 & $24 \mathrm{~h}$ & 18 & 1232 & $\mathrm{MD}-0.3(-0.69,0.09)$ & $\mathrm{I}^{2}=91 \%$ & Low \\
\hline \multirow{3}{*}{ Chen 2018} & $2 \mathrm{~h}$ & 2 & $143(71 / 72)$ & $\mathrm{MD}-0.45(-0.88,-0.02)$ & $\mathrm{I}^{2}=38 \%, p=0.20$ & \multirow{3}{*}{ Low } \\
\hline & $8 \mathrm{~h}$ & 2 & $143(71 / 72)$ & $\mathrm{MD}-0.62(-0.95,-0.28)$ & $\mathrm{I}^{2}=0 \%, p=0.69$ & \\
\hline & $24 \mathrm{~h}$ & 2 & $100(50 / 50)$ & $\mathrm{MD}-0.38(-0.79,0.02)$ & $\mathrm{I}^{2}=4 \%, p=0.31$ & \\
\hline \multirow{2}{*}{ Wang 2017} & At rest & 3 & $325(164 / 161)$ & ES $-1.206(-2.084,-0.329)$ & $\mathrm{I}^{2}=92.409, p<0.001$ & \multirow[b]{2}{*}{ Low } \\
\hline & At movement & 2 & $265(134 / 131)$ & ES $-1.435(-2.631,-0.240)$ & $\mathrm{I}^{2}=94.265, p<0.001$ & \\
\hline \multirow{6}{*}{ Zeng 2016} & Mg vs. placebo & & & & & \multirow{6}{*}{ Low } \\
\hline & 24 or $48 \mathrm{~h}$ & 5 & $289(145 / 144)$ & $\mathrm{MD}-0.41(-0.78,-0.05)$ & $\mathrm{I}^{2}=80 \%, p=0.0006$ & \\
\hline & $\mathrm{Mg}$ vs. bupi & & & & & \\
\hline & 24 or $48 \mathrm{~h}$ & 3 & $154(77 / 77)$ & MD $0.17(-0.92,1.26)$ & $\mathrm{I}^{2}=88 \%, p=0.0002$ & \\
\hline & $\mathrm{Mg}+$ bupi vs. bupi & & & & & \\
\hline & 18 or $24 \mathrm{~h}$ & 3 & $154(77 / 77)$ & $\mathrm{MD}-0.41(-0.87,0.04)$ & $\mathrm{I}^{2}=73 \%, p=0.03$ & \\
\hline \multirow[t]{2}{*}{ Guo 2015} & $\begin{array}{c}\text { At rest } \\
\text { total } \\
\text { At movement }\end{array}$ & \multirow[t]{2}{*}{ NR } & \multirow[t]{2}{*}{ NR } & SMD $-1.43(-2.74,-0.12)$ & $p<0.01$ & \multirow[t]{2}{*}{$\mathrm{CE}$} \\
\hline & $24 \mathrm{~h}$ & & & SMD $-0.05(-0.43,0.32)$ & NR & \\
\hline \multirow{6}{*}{ De Oliveira 2013} & At rest & & & & & \multirow{6}{*}{ Moderate } \\
\hline & Early $(0-4 \mathrm{~h})$ & 18 & $1153(567 / 586)$ & $\mathrm{MD}-0.74(-1.08,-0.48)$ & $\mathrm{I}^{2}=87 \%$ & \\
\hline & Late $(24 \mathrm{~h})$ & 13 & $606(302 / 304)$ & $\mathrm{MD}-0.36(-0.63,-0.09)$ & $\mathrm{I}^{2}=71 \%$ & \\
\hline & At movement & & & & & \\
\hline & Early $(0-4 \mathrm{~h})$ & 6 & $466(224 / 242)$ & MD $0.52(-1.15,0.10)$ & $\mathrm{I}^{2}=57 \%$ & \\
\hline & Late $(24 \mathrm{~h})$ & 5 & $285(142 / 143)$ & $\mathrm{MD}-0.73(-1.37,-0.1)$ & $\mathrm{I}^{2}=72 \%$ & \\
\hline \multirow{6}{*}{ Albrecht 2013} & At rest & & & & & \multirow{6}{*}{ Low } \\
\hline & Early & 15 & $868(433 / 435)$ & $\mathrm{MD}-6.9(-9.6,-4.2)$ & $\mathrm{I}^{2}=79 \%, p<0.00001$ & \\
\hline & $24 \mathrm{~h}$ & 14 & $900(434 / 466)$ & $\mathrm{MD}-4.2(-6.3,-2.1)$ & $\mathrm{I}^{2}=78 \%, p<0.00001$ & \\
\hline & At movement & & & & & \\
\hline & Early & 5 & $381(190 / 191)$ & $\mathrm{MD}-6.5(-10.0,-2.9)$ & $\mathrm{I}^{2}=78 \%, p=0.19$ & \\
\hline & $24 \mathrm{~h}$ & 5 & $225(112 / 113)$ & $\mathrm{MD}-9.2(-16.1,-2.3)$ & $\mathrm{I}^{2}=86 \%, p<0.00001$ & \\
\hline \multirow{2}{*}{ Murphy 2013} & $4-6 \mathrm{~h}$ & 16 & $956(477 / 479)$ & $\mathrm{MD}-0.67(-1.12,-0.23)$ & $\mathrm{I}^{2}=96 \%, p<0.00001$ & \multirow{2}{*}{ Low } \\
\hline & $20-24 \mathrm{~h}$ & 15 & $908(458 / 458)$ & $\mathrm{MD}-0.25(-0.62,0.71)$ & $\mathrm{I}^{2}=94 \%, p<0.00001$ & \\
\hline
\end{tabular}

$\mathrm{N}$, number; VAS, visual analogue scale; bupi, bupivacaine; NR, not reported; CE, can't evaluate; Mg, magnesium group; MD, mean difference; SMD, standardized mean difference; ES, effect size; CI, confidence interval. 
Ten studies conducted meta-analyses to evaluate the effect of magnesium on analgesic consumption [16,17,22-28,31]. All included studies showed a significant reduction in analgesic consumption after surgery when magnesium was administered, whereas the majority of included studies showed substantial heterogeneity. Summarized evidence on analgesic consumption is shown in Table 3.

Table 3. Summary of evidence: analgesic consumption (mg) in adult patients.

\begin{tabular}{|c|c|c|c|c|c|}
\hline First Author, Year & Study Number & $\begin{array}{l}\text { Participants Number } \\
\text { (Mg/Control) }\end{array}$ & MD, SMD, ES (95\% CI) & Heterogeneity & $\begin{array}{l}\text { Quality of Evidence } \\
\text { (GRADE) }\end{array}$ \\
\hline Shi 2021 & 8 & $449(229 / 220)$ & $\mathrm{MD}-4.23(-4.64,-3.82)$ & $\mathrm{I}^{2}=27 \%, p=0.21$ & High \\
\hline Ma 2021 & 5 & $290(145 / 145)$ & SMD $-3.20(-5.45,-0.95)$ & $\mathrm{I}^{2}=97.6 \%, p<0.001$ & Very low \\
\hline Li 2020 & 5 & $300(150 / 150)$ & SMD $-2.65(-4.23,-1.06)$ & $\mathrm{I}^{2}=96 \%, p<0.00001$ & Very low \\
\hline $\mathrm{Ng} 2020$ & 19 & 945 & $\mathrm{MD}-5.41(-7.08,-3.74)$ & $\mathrm{I}^{2}=92 \%, p<0.001$ & Low \\
\hline Chen 2018 & 2 & $143(71 / 72)$ & SMD $-0.40(-0.73,-0.07)$ & $\mathrm{I}^{2}=0 \%, p=0.57$ & Moderate \\
\hline Wang 2017 & 4 & 193/193 & ES $-1.620(-2.434,-0.806)$ & $\mathrm{I}^{2}=83.166 \%, p<0.001$ & Low \\
\hline Guo 2015 & NR & NR & SMD $-1.72(-3.21,-0.23)$ & NR & $\mathrm{CE}$ \\
\hline De Oliveira 2013 & 16 & $921(479 / 442)$ & MD $-10.52(-13.50,-7.54)$ & $\mathrm{I}^{2}=88 \%$ & Low \\
\hline Albrecht 2013 & 19 & $1054(527 / 527)$ & $\mathrm{MD}-7.6(-9.5,-5.8)$ & $\mathrm{I}^{2}=92 \%, p<0.00001$ & Low \\
\hline Murphy 2013 & 12 & $698(349 / 349)$ & MD $-7.40(-9.40,-5.41)$ & $\mathrm{I}^{2}=87 \%, p<0.00001$ & Low \\
\hline
\end{tabular}

$\mathrm{Mg}$, magnesium group; $\mathrm{MD}$, mean difference; SMD, standardized mean difference; $\mathrm{ES}$, effect size; $\mathrm{CI}$, confidence interval; NR, not reported; $\mathrm{CE}$, cannot evaluate.

Nine studies performed meta-analyses to assess the time interval to the first analgesic request following surgery $[17,22,24,27-32]$. Time to first analgesic request was significantly shorter in the majority of studies although heterogeneity was substantial; the details are presented in Table 4.

Table 4. Summary of evidence: time to first analgesic request (min) in adult patients.

\begin{tabular}{|c|c|c|c|c|c|}
\hline First Author, Year & Study Number & $\begin{array}{l}\text { Participants Number } \\
\text { (Mg/Control) }\end{array}$ & MD, SMD, RoM (95\% CI) & Heterogeneity & $\begin{array}{l}\text { Quality of Evidence } \\
\text { (GRADE) }\end{array}$ \\
\hline Shi 2021 & 11 & $613(311 / 302)$ & MD, $329.99(228.73,431.24)$ & $\mathrm{I}^{2}=99 \%, p<0.00001$ & Low \\
\hline Ma 2021 & 8 & $880(440 / 440)$ & SMD,$-3.0 .(-4.32,-1.74)$ & $\mathrm{I}^{2}=96.3 \%, p<0.001$ & Low \\
\hline Li 2020 & 6 & $400(200 / 200)$ & SMD, $4.96(2.75,7.17)$ & $\mathrm{I}^{2}=98 \%, p<0.00001$ & Very low \\
\hline Ng 2020 & 11 & 824 & MD, $143(103,183)$ & $\mathrm{I}^{2}=99 \%, p<0.001$ & Low \\
\hline Wang 2020 & 9 & $660(330 / 330)$ & RoM, $1.23(1.13,1.33)$ & $\mathrm{I}^{2}=96 \%, p<0.00001$ & Low \\
\hline Zeng 2016 & $\begin{array}{c}4 \text { (Mg vs. placebo) } \\
3 \text { (Mg vs. bupi) } \\
3 \text { (Mg + bupi vs. } \\
\text { bupi) }\end{array}$ & $\begin{array}{c}229(115 / 114) \\
154(77 / 77) \\
154(77 / 77)\end{array}$ & $\begin{array}{c}\mathrm{MD}, 3.59(0.26,6.93) \\
\mathrm{MD},-0.82(-5.83,4.20) \\
\mathrm{MD}, 6.25(5.22,7.29)\end{array}$ & $\begin{array}{c}\mathrm{I}^{2}=99 \%, p<0.00001 \\
\mathrm{I}^{2}=99 \%, p<0.00001 \\
\mathrm{I}^{2}=69 \%, p=0.04\end{array}$ & Low \\
\hline De Oliveira 2013 & 4 & $339(161 / 178)$ & $\mathrm{MD}, 4.4(-6.9,15.9)$ & NR & $\mathrm{CE}$ \\
\hline Albrecht 2013 & 4 & $298(149 / 149)$ & MD, $7.2(-1.9,16.2)$ & $\mathrm{I}^{2}=90 \%, p<0.00001$ & Low \\
\hline Pascual-Ramirez 2013 & 10 & NR & MD, 85 SMD, $0.98(0.51,1.37)$ & $\mathrm{I}^{2}=56 \%, p<0.001$ & Moderate \\
\hline
\end{tabular}

$\mathrm{Mg}$, magnesium group; Bupi, bupivacaine; MD, mean difference; SMD, standardized mean difference; RoM, ratio of means; CI, confidence interval; NR, not reported; CE, can't evaluate.

In pediatric patients, the pain score did not show any significant difference between the two groups. Two studies performed meta-analyses on the incidence of rescue analgesics $[19,20]$, which was significantly lower in patients who received magnesium. Summarized evidence on pain-related outcomes in pediatric patients is shown in Table 5.

\subsection{Confidence and Quality of Evidence}

Based on the AMSTAR 2.0 tool, the confidence of the included systematic reviews was deemed low to critically low. The quality of evidence on pain-related outcomes ranged from very low to moderate according to the GRADE system, with a large body of lowquality evidence. We assessed the quality of evidence on pain-related outcomes provided by each systematic review if there was no GRADE evaluation. They are summarized in Tables $2-5$ for each outcome and patient category. 
Table 5. Summary of evidence in pediatric patients.

\begin{tabular}{|c|c|c|c|c|c|c|}
\hline First Author, Year & Outcome & Study N & $\begin{array}{l}\text { Participant N } \\
\text { (Mg/Control) }\end{array}$ & RR, SMD, MD (95\% CI) & Heterogeneity & $\begin{array}{c}\text { Quality of } \\
\text { Evidence (GRADE) }\end{array}$ \\
\hline Kawakami 2018 & $\begin{array}{l}\text { Incidence of rescue } \\
\text { analgesia }\end{array}$ & 4 & $247(117 / 130)$ & RR $0.45(0.24,0.86)$ & $\mathrm{I}^{2}=62.5 \%, p=0.046$ & Very low \\
\hline Cho 2017 & $\begin{array}{l}\text { Pain score } \\
15 \mathrm{~min} \\
1 \mathrm{~h} \\
24 \mathrm{~h}\end{array}$ & $\begin{array}{l}6 \\
9 \\
6\end{array}$ & $\begin{array}{l}405(203 / 202) \\
615(308 / 307) \\
330(165 / 165)\end{array}$ & $\begin{array}{c}\text { SMD }-0.26(-0.52,0.00) \\
\text { SMD } 0.05(-0.70,0.80) \\
\text { SMD }-0.39(-0.71,-0.07)\end{array}$ & $\begin{array}{l}\mathrm{I}^{2}=40.36 \%, p=0.1232 \\
\mathrm{I}^{2}=94.94 \%, p<0.0001 \\
\mathrm{I}^{2}=50.56 \%, p=0.0727\end{array}$ & Low \\
\hline \multirow[t]{2}{*}{ Xie 2017} & $\begin{array}{c}\text { Pain score } \\
\text { (mCHEOPs) } \\
15 \mathrm{~min} \\
1 \mathrm{~h}\end{array}$ & $\begin{array}{l}2 \\
2\end{array}$ & $\begin{array}{l}160(80 / 80) \\
160(80 / 80)\end{array}$ & $\begin{array}{c}\text { MD } 0.17(-0.02,0.35) \\
M D-0.59(-3.11,1.93)\end{array}$ & $\begin{array}{c}\mathrm{I}^{2}=0 \%, p=0.77 \\
\mathrm{I}^{2}=98 \%, p<0.00001\end{array}$ & Low \\
\hline & $\begin{array}{c}\text { Incidence of rescue } \\
\text { analgesia }\end{array}$ & 5 & $305(153 / 152)$ & RR $0.53(0.31,0.91)$ & $\mathrm{I}^{2}=69 \%, p=0.01$ & Low \\
\hline
\end{tabular}

$\mathrm{N}$, number; RR, risk ratio; SMD, standardized mean difference; $\mathrm{MD}$, mean difference; CI, confidence interval; mCHEOPs, (modifiedChildren's Hospital of Eastern Ontario Pain scale).

\subsection{Results of Updated Meta-Analysis}

Data of adult patients from 109 RCTs (of which two RCTs were newly included in this updated meta-analysis [33,34], and data of pediatric patients from 13 RCTs were extracted (supplementary Figure S1). In adult patients, pain scores at the PACU, early phase, and late phase showed more significant reductions in the magnesium group than in the control group (Table 6). The time to first analgesic was significantly longer in the magnesium group than in the control group (SMD $=-1.867 ; 95 \% \mathrm{CI},-2.216$ to $-1.519 ; p_{\mathrm{chi}}{ }^{2}<0.001$; $\mathrm{I}^{2}=94.8 \%$ ). Analgesic consumption was significantly reduced with the use of magnesium compared to the control group (SMD $=1.456 ; 95 \% \mathrm{CI}, 1.163-1.749 ; p_{\text {chi }}{ }^{2}<0.001 ; \mathrm{I}^{2}=94.7 \%$ ).

Table 6. Pain score results of updated meta-analysis and TSA in adult patients.

\begin{tabular}{|c|c|c|c|}
\hline Post & tive Pain Score & $\begin{array}{l}\text { Quantitative Meta-Analysis } \\
\text { (SMD; } 95 \% \mathrm{CI} ; p_{\mathrm{chi}}{ }^{2} ; \mathrm{I}^{2} \text { ) }\end{array}$ & Description of Trial Sequential Analysis (TSA) \\
\hline \multirow{2}{*}{ PACU } & Rest $(0-1 \mathrm{~h})$ & $0.395 ; 0.178-0.612 ;<0.001 ; 85.9 \%$ & $\begin{array}{l}\text { Pain at rest }(0 \mathrm{~h}) \text { : TSA indicated that } 98.7 \% \text { ( } 2487 \text { of } 2520 \text { patients) of the RIS was } \\
\text { accrued. The cumulative } Z \text { curve crossed both the conventional test boundary and } \\
\text { the trial sequential monitoring boundary. }\end{array}$ \\
\hline & Movement $(0-1 \mathrm{~h})$ & $\begin{array}{l}0.437 ;-0.113-0.988 ;<0.001 ; 88.3 \% \\
0.485 ;-0.275-1.245 ;<0.001 ; 89.5 \%\end{array}$ & $\begin{array}{l}\text { Pain at movement }(0 \mathrm{~h}) \text { : The trial sequential monitoring boundary was ignored due } \\
\text { to too little information use. The cumulative } Z \text { curve did not cross the conventional } \\
\text { test boundary. }\end{array}$ \\
\hline \multirow{2}{*}{$\begin{array}{l}\text { Early } \\
\text { phase }\end{array}$} & Rest $(0-4$ h) & $0.872 ; 0.638-1.106 ;<0.001 ; 91.2 \%$ & $\begin{array}{l}\text { Pain at rest }(4 \mathrm{~h}) \text { : TSA indicated that accrued number of patients ( } 3830) \text { exceed the } \\
\text { RIS (2959). The cumulative } Z \text { curve crossed both the conventional test boundary and } \\
\text { the trial sequential monitoring boundary. }\end{array}$ \\
\hline & Movement $(0-4 \mathrm{~h})$ & $1.059 ; 0.561-1.556 ;<0.001 ; 89.6 \%$ & $\begin{array}{l}\text { Pain at movement }(4 \mathrm{~h}) \text { : TSA indicated that } 89.0 \% \text { ( } 832 \text { of } 934 \text { patients) of the RIS was } \\
\text { accrued. The cumulative } Z \text { curve crossed both the conventional test boundary and } \\
\text { the trial sequential monitoring boundary. }\end{array}$ \\
\hline \multirow{2}{*}{$\begin{array}{l}\text { Late } \\
\text { phase }\end{array}$} & Rest (24 h) & $0.470 ; 0.307-0.633 ;<0.001 ; 81.6 \%$ & $\begin{array}{l}\text { Pain at rest }(24 \mathrm{~h}) \text { : TSA indicated that accrued number of patients }(3500) \text { exceed the } \\
\text { RIS ( } 3115) \text {. The cumulative } Z \text { curve crossed both the conventional test boundary and } \\
\text { the trial sequential monitoring boundary. }\end{array}$ \\
\hline & Movement $(24 \mathrm{~h})$ & $0.679 ; 0.388-0.970 ;<0.001 ; 61.1 \%$ & $\begin{array}{l}\text { Pain at movement }(24 \mathrm{~h}) \text { : TSA indicated that only } 60.8 \% \text { ( } 507 \text { of } 834 \text { patients) of the } \\
\text { RIS was accrued. The cumulative } Z \text { curve crossed both the conventional test } \\
\text { boundary and the trial sequential monitoring boundary. }\end{array}$ \\
\hline
\end{tabular}

PACU, post-anesthesia care unit; SMD, standardized mean difference; RIS, required information size.

In pediatric patients, pain scores at the PACU and early phase showed significant reductions in the magnesium group compared to the control group, whereas the pain score at the late phase (at postoperative $24 \mathrm{~h}$ ) did not show a significant difference between the two groups (Table 7). The time to first analgesic was significantly longer in the magnesium group than in the control group, and analgesic consumption and the incidence of rescue were significantly lower in the magnesium group than in the control group (Table 7). Forest plots of pain score at postoperative $4 \mathrm{~h}$ in adult patients and the incidence of rescue analgesic in pediatric patients are presented in Figures 2 and 3, respectively. The other forest plots are shown in supplementary figures: adult resting pain score at $0 \mathrm{~h}, 0-1 \mathrm{~h}$, $0-4 \mathrm{~h}$, and $24 \mathrm{~h}$ in Figures S2-S5; adult dynamic pain score at $0 \mathrm{~h}, 0-1 \mathrm{~h}, 4 \mathrm{~h}, 0-4 \mathrm{~h}$, and $24 \mathrm{~h}$ 
in Figures S6-S10; pediatric pain score at $0 \mathrm{~h}, 0-1 \mathrm{~h}, 4 \mathrm{~h}, 0-4 \mathrm{~h}$, and $24 \mathrm{~h}$ in Figures S11-S15; and pediatric time to first analgesic and analgesic consumption in Figures S16 and S17.

Table 7. Pain-related outcomes of updated meta-analysis and TSA in pediatric patients.

\begin{tabular}{|c|c|c|c|c|}
\hline & Postoperative Outcomes & & $\begin{array}{l}\text { Quantitative Meta-Analysis } \\
\text { (SMD or RR; } 95 \% \text { CI; } p_{\mathrm{chi}^{2}}{ }^{2} \mathrm{I}^{2} \text { ) }\end{array}$ & Description of Trial Sequential Analysis (TSA) \\
\hline \multirow{5}{*}{ Pain score } & \multirow[b]{2}{*}{ PACU } & $0 \mathrm{~h}$ & $0.811 ; 0.194-1.429 ;<0.001 ; 94.2 \%$ & \multirow{2}{*}{$\begin{array}{l}\text { Pain }(0 \mathrm{~h}) \text { : TSA indicated that only } 12.6 \% \text { ( } 853 \text { of } 6776 \text { patients) of } \\
\text { the RIS was accrued. The cumulative } Z \text { curve crossed the } \\
\text { conventional test boundary but returned within the conventional } \\
\text { boundary during TSA. }\end{array}$} \\
\hline & & $0-1 \mathrm{~h}$ & $0.553 ; 0.065-1.040 ;<0.001 ; 90.7 \%$ & \\
\hline & \multirow[t]{2}{*}{ Early phase } & $4 \mathrm{~h}$ & $0.536 ; 0.064-1.008 ;<0.001 ; 82.4 \%$ & \multirow{2}{*}{$\begin{array}{l}\text { Pain }(4 \mathrm{~h}) \text { : The trial sequential monitoring boundary was ignored } \\
\text { due to too little information use. The cumulative } Z \text { curve crossed } \\
\text { the conventional test boundary but did not cross the trial } \\
\text { sequential monitoring boundary. }\end{array}$} \\
\hline & & $0-4 \mathrm{~h}$ & $0.452 ;-0.010-0.914 ;<0.001 ; 89.7 \%$ & \\
\hline & Late phase & $24 \mathrm{~h}$ & $0.342 ;-0.360-1.044 ;<0.001 ; 93.8 \%$ & $\begin{array}{l}\text { Pain }(24 \mathrm{~h}) \text { : The trial sequential monitoring boundary was } \\
\text { ignored due to too little information use. The cumulative } Z \text { curve } \\
\text { did not cross the conventional test boundary. }\end{array}$ \\
\hline \multicolumn{3}{|c|}{ Time to first analgesic } & $-1.222 ;-2.345-0.098 ;<0.001 ; 92.4 \%$ & $\begin{array}{l}\text { The trial sequential monitoring boundary was ignored due to too } \\
\text { little information use. The cumulative } Z \text { curve crossed the } \\
\text { conventional test boundary but did not cross the trial sequential } \\
\text { monitoring boundary. }\end{array}$ \\
\hline \multicolumn{3}{|c|}{ Analgesic consumption } & $1.144 ; 0.370-1.917 ;<0.001 ; 88.8 \%$ & $\begin{array}{l}\text { TSA indicated that only } 10.1 \% \text { ( } 292 \text { of } 2881 \text { patients) of the RIS } \\
\text { was accrued. The cumulative } Z \text { curve crossed the conventional } \\
\text { test boundary but did not cross the trial sequential monitoring } \\
\text { boundary. }\end{array}$ \\
\hline \multicolumn{3}{|c|}{$\begin{array}{l}\text { Incidence } \\
\text { of rescue analgesic }\end{array}$} & $1.991 * ; 1.385-2.862 ; 0.014 ; 58.2 \%$ & $\begin{array}{l}\text { TSA indicated that only } 80.8 \% \text { ( } 552 \text { of } 683 \text { patients) of the RIS was } \\
\text { accrued. The cumulative } Z \text { curve crossed both the conventional } \\
\text { test boundary and the trial sequential monitoring boundary. }\end{array}$ \\
\hline
\end{tabular}

PACU, post-anesthesia care unit; SMD, standardized mean difference; RR, risk ratio; CI, confidence interval; RIS, required information size. $*$ RR.

TSA suggested that results of our updated meta-analysis were confirmative for each outcome (Tables 6 and 7). Figure 4 shows the results of TSA of resting pain score at postoperative $4 \mathrm{~h}$ in adult patients. The other parameters with respect to the adult resting pain score at $0 \mathrm{~h}$ and $24 \mathrm{~h}$ are shown in Figures S18 and S19; adult dynamic pain score at $0 \mathrm{~h}$, $4 \mathrm{~h}$, and $24 \mathrm{~h}$ in Figures S20-S22; pediatric pain score at $0 \mathrm{~h}, 4 \mathrm{~h}$, and $24 \mathrm{~h}$ in Figures S23-S25; and time to first analgesic, analgesic consumption, and incidence of rescue analgesic in Figures S26-S28.

\subsection{Publication Bias}

A funnel plot was used for all comparisons. All displayed a symmetrical appearance for resting pain score at $0 \mathrm{~h}, 0-1 \mathrm{~h}, 4 \mathrm{~h}, 0-4 \mathrm{~h}$, and $24 \mathrm{~h}$ (Figures S29-S33); adult dynamic pain score at $0 \mathrm{~h}, 0-1 \mathrm{~h}, 4 \mathrm{~h}, 0-4 \mathrm{~h}$, and $24 \mathrm{~h}$ (Figures S34-S38); time to first analgesic (Figure S39) and analgesic consumption (Figure S40) in adults; and pediatric pain score at $0 \mathrm{~h}, 0-1 \mathrm{~h}, 4 \mathrm{~h}, 0-4 \mathrm{~h}$, and $24 \mathrm{~h}$ (Figures S41-S45). The results of Egger's test were as follows: adult resting pain score at $0 \mathrm{~h}(p=0.170), 0-1 \mathrm{~h}(p=0.266), 4 \mathrm{~h}(p=0.212), 0-4 \mathrm{~h}$ $(p=0.103)$, and $24 \mathrm{~h}(p=0.834)$; adult dynamic pain score at $0 \mathrm{~h}(p=0.707), 0-1 \mathrm{~h}(p=0.308)$, $4 \mathrm{~h}(p=0.066), 0-4 \mathrm{~h}(p=0.002)$, and $24 \mathrm{~h}(p=0.064)$, time to first analgesic use $(p<0.001)$ in adults; analgesic consumption $(p<0.001)$ in adults; and pediatric pain score at $0 \mathrm{~h}$ $(p=0.114), 0-1 \mathrm{~h}(p=0.235), 4 \mathrm{~h}(p=0.734), 0-4 \mathrm{~h}(p=0.175)$, and $24 \mathrm{~h}(p=0.696)$.

In terms of outcomes with $p$-value $<0.1$ from Egger's test, a trim-and-fill analysis was performed: adult dynamic pain score at $4 \mathrm{~h}(\mathrm{SMD}=0.942 ; 95 \% \mathrm{CI}, 0.364$ to $1.520)$ and at $0-4 \mathrm{~h}(\mathrm{SMD}=1.059 ; 95 \% \mathrm{CI}, 0.561$ to 1.556$)$ and analgesic consumption in adults (SMD $=-1.456 ; 95 \% \mathrm{CI}, 1.163$ to 1.749 ) showed significant changes after trim-andfill analysis ( $\mathrm{SMD}=0.536$; $95 \% \mathrm{CI},-0.120$ to 1.191 ; $\mathrm{SMD}=0.470 ; 95 \% \mathrm{CI},-0.082$ to 1.022 ; $\mathrm{SMD}=0.539 ; 95 \% \mathrm{CI}, 0.226$ to 0.852 , respectively). 


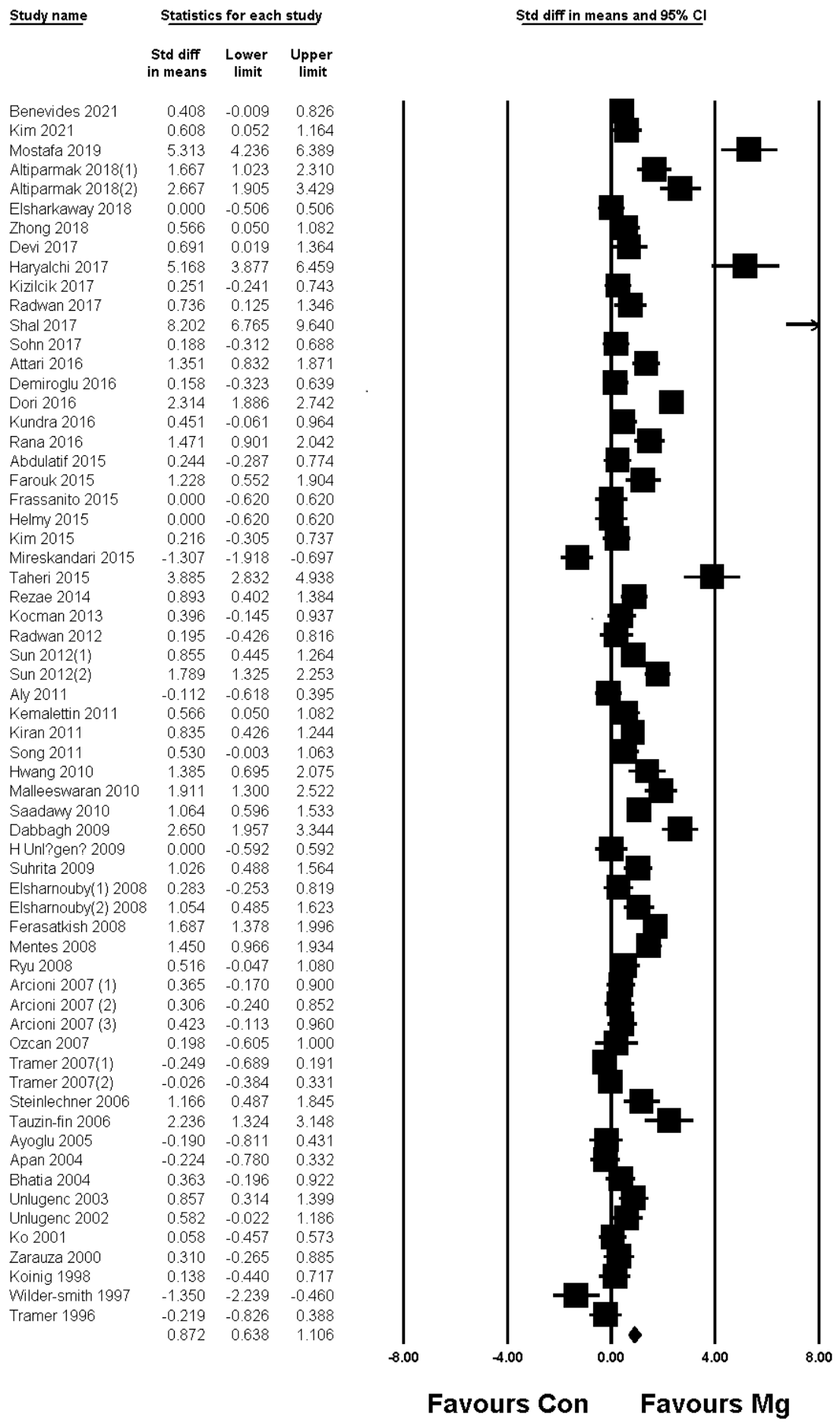

Figure 2. Forest plot showing pain score at postoperative $4 \mathrm{~h}$ in adult patients. The figure depicts individual trials as filled squares with relative sample size and the $95 \%$ confidence interval (CI) of the difference as a solid line. The diamond shape indicates the pooled estimate and uncertainty for the combined effect. 
Study name

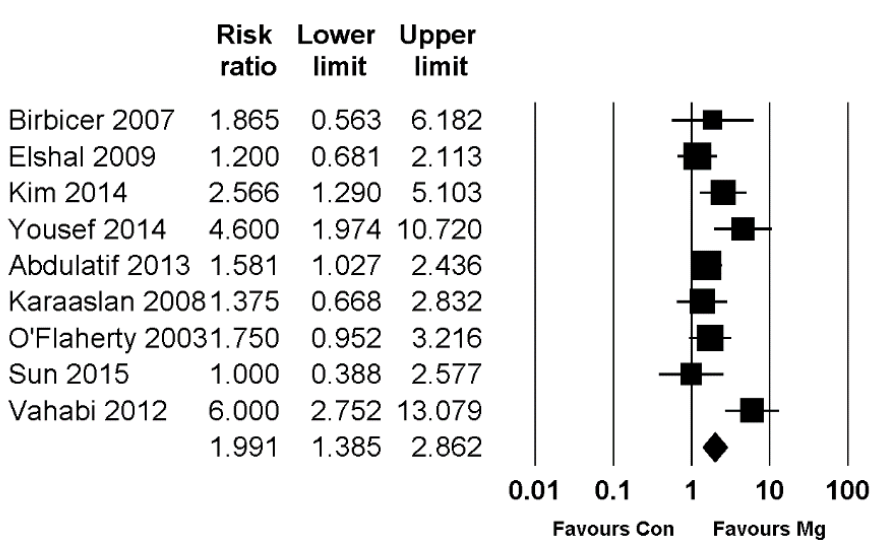

Figure 3. Forest plot showing the incidence of rescue analgesic in pediatric patients. The figure depicts individual trials as filled squares with relative sample size and the $95 \%$ confidence interval (CI) of the difference as a solid line. The diamond shape indicates the pooled estimate and uncertainty for the combined effect.

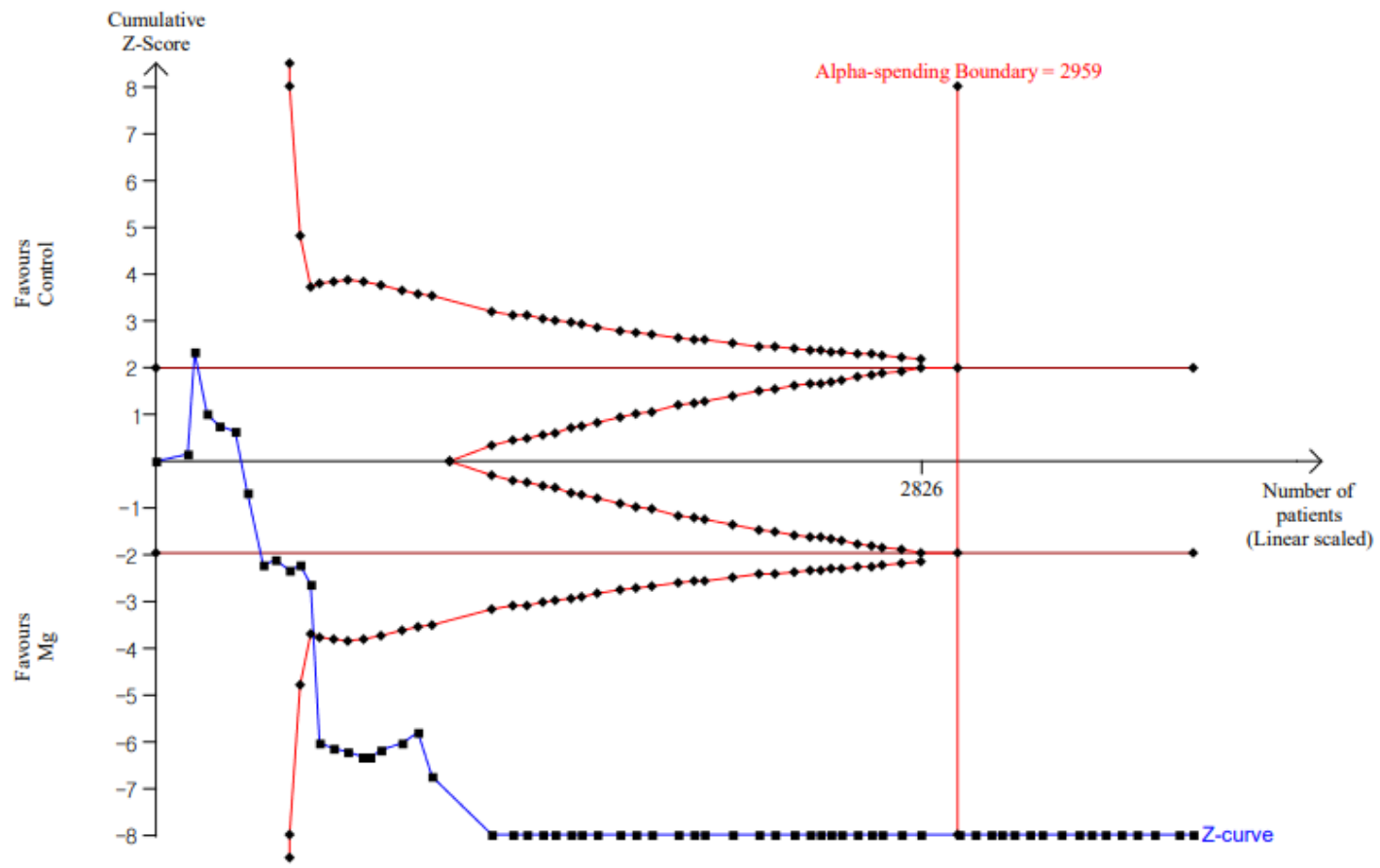

Figure 4. TSA for resting pain score at postoperative $4 \mathrm{~h}$ in adult patients. Complete blue line represents the cumulative $\mathrm{Z}$ curve, etched red line represents conventional test boundary, complete outer red line represents the trial sequential monitoring boundary, and complete inner red line represents futility boundary. The cumulative $Z$ curve crossed the conventional test boundary and the trial sequential monitoring boundary.

\section{Discussion}

Through this study, we provided a comprehensive overview of the reported effect of perioperative administration of magnesium on postoperative pain by incorporating evidence from meta-analyses and systematic reviews of RCTs and conducting an updated meta-analysis with TSA. The overall quality of evidence ranged from very low to moderate. We discovered that there was a large body of low-quality evidence that perioperative magnesium administration reduced the intensity of postoperative pain and diminished the need for postoperative opioid analgesia. In contrast, there is a small body of high-quality evidence supporting postoperative pain-related outcomes. Individual systematic reviews 
of this umbrella review included only RCTs as study designs. RCTs are initially given a high-quality rating, but after considering study limitations, indirectness, inconsistency, imprecision, and publication bias, they might be downgraded [35]. The possibility of bias and inconsistency can explain the current study's predominantly low quality of evaluated outcomes. Various regimens, dosages, routes of magnesium administration, and different types of surgery can all have an impact on the quality of evidence based on the GRADE system.

An updated meta-analysis suggested that perioperative magnesium administration showed a significantly beneficial effect on postoperative analgesia, even with substantial heterogeneity. Indeed, as our understanding of the component of this receptor linked with pain pathophysiology has grown, the number of trials on systemic or local usage of magnesium has expanded in recent years. Magnesium modulates pain and inflammatory responses by blocking calcium channels and antagonizing the NMDA receptor, thereby reducing central sensitization to peripheral injury [5]. Moreover, there has been a growing interest in multimodal analgesia as an important component of appropriate perioperative pain-management strategy in patients undergoing surgery. Multimodal analgesia is defined as the use of various agents, primarily non-opioid analgesics, and non-pharmacologic therapies in combination to target a range of pain receptors in a synergistic manner [36]. Given its analgesic properties, magnesium can be a good option for multimodal analgesia. By assessing the overall quality and quantity of existing evidence in perioperative magnesium use for postoperative analgesia in this study, we may expect to narrow the gap between study evidence and its clinical applicability.

The findings of several recent trials regarding the use of perioperative magnesium are reassuring and have demonstrated the analgesic properties of magnesium in pediatric patients $[18,20]$. Unlike in adult trials, perioperative magnesium had no beneficial effect on pain intensity in pediatric patients in the current study. It is difficult to assess pain in children because they are unable or unwilling to express it. Even the Children's Hospital of Eastern Ontario Pain Scale (CHEOPS), a pain rating system, is questionable because the ratings are often low and do not correspond well with self-reported measures [37]. In contrast, the considerable reduction in the incidence of rescue analgesia mediated by magnesium administration in this study may objectively suggest that perioperative magnesium administration can be useful in the pediatric group.

The overall confidence of the included systematic reviews was rated as critically low to low using AMSTAR 2.0 tool. The quality of evidence on pain-related outcomes reported in included studies ranged from very low to moderate based on GRADE system. Because of its multifaceted and subjective nature, pain is a complex clinical phenomena to quantify [38]. There is a general lack of uniformity in pain-related clinical trials, both regarding pain-related outcome assessment and description, making it difficult to synthesize data [39]. In this respect, the accuracy and practicality of objective pain measurements are advantageous. Analgesic consumption and incidence or time to first analgesia can be useful parameters as objective pain measurement tools in this regard, even if these outcomes exhibit significant heterogeneity. The analgesic effect of perioperative magnesium administration was examined in our study using subjective and objective measures, which could support its use for analgesic benefit. However, we should regard the low quality of evidence based on our assessment in this study, which means that more research will almost certainly have a considerable impact on confidence in the estimate of the effect and will certainly change the estimate. Given the inherent subjectivity of the GRADE approach, we performed TSA in addition to an updated meta-analysis. TSA can be thought of as a purely objective and quantitative approach [40], with the results indicating that current outcomes from existing research are sufficient and that therefore additional research may not be required. However, because sequential approaches have methodological limitations when heterogeneity is present, this result cannot be considered a powered conclusion [41]. When building on existing databases, TSA should be treated with caution because omissions and errors in interpretation and implementation may be common [42,43]. Hence, 
further research on the ideal amount of information and different types of interventions under diverse circumstances is needed to draw appropriate conclusions.

Given the wide variability in the methodology used in the included trials, this umbrella review has some limitations. Various surgical methods and anesthetic strategies were addressed in all the included studies. Although this is a standard method for quantitative systematic reviews of perioperative pain management, it is possible that this contributed to some of the observed heterogeneity. The routes of magnesium administration varied according to the anesthetic approach, contributing to high heterogeneity. This allowed for a more precise assessment of the magnesium analgesic effect, but it also limits the applicability of our findings to anesthetic practice when multimodal drugs are frequently used. Furthermore, in this study, we did not assess the safety of perioperative magnesium use, such as shivering, nausea, or vomiting following surgery. This will provide more information on the perioperative use of magnesium. Despite these limitations, our study has shown strength by including RCTs to present the first umbrella review of the evidence on the effectiveness of perioperative magnesium as an adjuvant for postoperative analgesia.

\section{Conclusions}

While most of included studies demonstrated a statistically significant improvement in outcomes related to postoperative pain when systemic magnesium was administered during the perioperative period, current findings reveal that our confidence in the beneficial effect of perioperative magnesium on postoperative pain is limited. While the updated meta-analysis with TSA suggested that the existing evidence is sufficient, additional research is needed to confirm the objective efficacy of perioperative magnesium for postoperative analgesia as well as research with reliable assessment of potential biases and appropriate interpretation of heterogeneity. Further studies applying standardized definitions of outcomes and magnesium administration protocols for evaluating postoperative pain could present more exact estimates, decrease the risk of heterogeneity, and increase reliability.

Supplementary Materials: The following are available online at https://www.mdpi.com/article/10 $.3390 / j p m 11121273 /$ s1, Figure S1: Flow diagram of updated meta-analysis; Figure S2: Forest plot showing resting pain score at postoperative $0 \mathrm{~h}$ in adult patients; Figure S3: Forest plot showing resting pain score at postoperative $0-1 \mathrm{~h}$ in adult patients; Figure S4: Forest plot showing resting pain score at postoperative $0-4 \mathrm{~h}$ in adult patients; Figure S5: Forest plot showing resting pain score at postoperative $24 \mathrm{~h}$ in adult patients; Figure S6: Forest plot showing dynamic pain score at postoperative $0 \mathrm{~h}$ in adult patients; Figure S7: Forest plot showing dynamic pain score at postoperative $0-1 \mathrm{~h}$ in adult patients; Figure S8: Forest plot showing dynamic pain score at postoperative $4 \mathrm{~h}$ in adult patients; Figure S9: Forest plot showing dynamic pain score at postoperative $0-4 \mathrm{~h}$ in adult patients; Figure S10: Forest plot showing dynamic pain score at postoperative $24 \mathrm{~h}$ in adult patients; Figure S11: Forest plot showing pain score at postoperative $0 \mathrm{~h}$ in pediatric patients; Figure S12: Forest plot showing pain score at postoperative $0-1 \mathrm{~h}$ in pediatric patients; Figure S13: Forest plot showing pain score at postoperative $4 \mathrm{~h}$ in pediatric patients; Figure S14: Forest plot showing pain score at postoperative $0-4 \mathrm{~h}$ in pediatric patients; Figure S15: Forest plot showing pain score at postoperative $24 \mathrm{~h}$ in pediatric patients; Figure S16: Forest plot showing time to first analgesic in pediatric patients; Figure S17: Forest plot showing analgesic consumption in pediatric patients; Figure S18: TSA for resting pain score at postoperative $0 \mathrm{~h}$ in adult patients; Figure S19: TSA for resting pain score at postoperative $24 \mathrm{~h}$ in adult patients; Figure S20: TSA for dynamic pain score at postoperative $0 \mathrm{~h}$ in adult patients; Figure S21: TSA for dynamic pain score at postoperative $4 \mathrm{~h}$ in adult patients; Figure S22: TSA for dynamic pain score at postoperative $24 \mathrm{~h}$ in adult patients; Figure S23: TSA for pain score at postoperative $0 \mathrm{~h}$ in pediatric patients; Figure S24: TSA for pain score at postoperative $4 \mathrm{~h}$ in pediatric patients; Figure S25: TSA for pain score at postoperative $24 \mathrm{~h}$ in pediatric patients; Figure S26: TSA for the time to first analgesic in pediatric patients; Figure S27: TSA for the analgesic consumption in pediatric patients; Figure S28: TSA for the incidence of rescue analgesic in pediatric patients; Figure S29: Funnel plot for resting pain score at postoperative $0 \mathrm{~h}$ in adult patients; Figure S30: Funnel plot for resting pain score at postoperative $0-1 \mathrm{~h}$ in adult patients; Figure S31: Funnel plot for resting pain score at postoperative $4 \mathrm{~h}$ in adult patients; Figure S32: 
Funnel plot for resting pain score at postoperative 0-4 h in adult patients; Figure S33: Funnel plot for resting pain score at postoperative $24 \mathrm{~h}$ in adult patients; Figure S34: Funnel plot for dynamic pain score at postoperative $0 \mathrm{~h}$ in adult patients; Figure S35: Funnel plot for dynamic pain score at postoperative $0-1 \mathrm{~h}$ in adult patients; Figure S36: Funnel plot for dynamic pain score at postoperative $4 \mathrm{~h}$ in adult patients; Figure S37: Funnel plot for dynamic pain score at postoperative $0-4 \mathrm{~h}$ in adult patients; Figure S38: Funnel plot for dynamic pain score at postoperative $24 \mathrm{~h}$ in adult patients; Figure S39: Funnel plot for the time to first analgesic in adult patients; Figure S40: Funnel plot for the analgesic consumption in adult patients; Figure S41: Funnel plot for pain score at postoperative $0 \mathrm{~h}$ in pediatric patients; Figure S42: Funnel plot for pain score at postoperative $0-1 \mathrm{~h}$ in pediatric patients; Figure S43: Funnel plot for pain score at postoperative $4 \mathrm{~h}$ in pediatric patients; Figure S44: Funnel plot for pain score at postoperative $0-4 \mathrm{~h}$ in pediatric patients; Figure S45: Funnel plot for pain score at postoperative $24 \mathrm{~h}$ in pediatric patients; File S1: PRISMA checklist; File S2: search term of umbrella review; File S3: search term of updated meta-analysis

Author Contributions: Conceptualization, G.J.C. and H.K.; methodology, G.J.C. and H.K.; software, H.K.; validation, G.J.C., H.-C.O. and H.K.; formal analysis, H.K.; investigation, G.J.C., Y.I.K. and Y.H.K.; resources, G.J.C. and Y.I.K.; data curation, Y.I.K. and Y.H.K.; writing-original draft preparation, G.J.C. and Y.I.K.; writing-review and editing, H.-C.O. and H.K.; visualization, G.J.C. and H.K.; supervision, H.K.; project administration, H.K.; funding acquisition, H.K. All authors have read and agreed to the published version of the manuscript.

Funding: This research was supported by the Basic Science Research Program through the National Research Foundation of Korea (NRF) funded by the Ministry of Education, Science and Technology [Grant No. NRF-2020R1C1C1011263]. The Funding bodies play no role in the design of the study and collection, analysis, and interpretation of data and in writing the manuscript.

Institutional Review Board Statement: Not applicable.

Informed Consent Statement: Not applicable.

Data Availability Statement: The datasets used and analyzed during the current study are available from the corresponding author upon reasonable request.

Acknowledgments: Not applicable.

Conflicts of Interest: The authors declare no conflict of interest. The funders had no role in the design of the study; in the collection, analyses, or interpretation of data; in the writing of the manuscript, or in the decision to publish the results.

\section{References}

1. Gan, T.J. Poorly controlled postoperative pain: Prevalence, consequences, and prevention. J. Pain Res. 2017, 10, 2287-2298. [CrossRef] [PubMed]

2. Buvanendran, A.; Kroin, J.S. Multimodal analgesia for controlling acute postoperative pain. Curr. Opin. Anaesthesiol. 2009, 22, 588-593. [CrossRef] [PubMed]

3. Bruhn, J.; Scheffer, G.J.; van Geffen, G.J. Clinical application of perioperative multimodal analgesia. Curr. Opin. Supportive Palliat. Care 2017, 11, 106-111. [CrossRef]

4. Tramer, M.R.; Schneider, J.; Marti, R.A.; Rifat, K. Role of magnesium sulfate in postoperative analgesia. Anesthesiology 1996, 84, 340-347. [CrossRef] [PubMed]

5. Fawcett, W.J.; Haxby, E.J.; Male, D.A. Magnesium: Physiology and pharmacology. Br. J. Anaesth. 1999, 83, 302-320.

6. McCarthy, R.J.; Kroin, J.S.; Tuman, K.J.; Penn, R.D.; Ivankovich, A.D. Antinociceptive potentiation and attenuation of tolerance by intrathecal co-infusion of magnesium sulfate and morphine in rats. Anesth. Analg. 1998, 86, 830-836. [PubMed]

7. Aromataris, E.; Fernandez, R.; Godfrey, C.M.; Holly, C.; Khalil, H.; Tungpunkom, P. Summarizing systematic reviews: Methodological development, conduct and reporting of an umbrella review approach. Int. J. Evid.-Based Healthc. 2015, 13, 132-140. [CrossRef] [PubMed]

8. Page, M.J.; McKenzie, J.E.; Bossuyt, P.M.; Boutron, I.; Hoffmann, T.C.; Mulrow, C.D.; Shamseer, L.; Tetzlaff, J.M.; Akl, E.A.; Brennan, S.E.; et al. The PRISMA 2020 statement: An updated guideline for reporting systematic reviews. BMJ 2021, 372 , n71. [CrossRef] [PubMed]

9. Shea, B.J.; Grimshaw, J.M.; Wells, G.A.; Boers, M.; Andersson, N.; Hamel, C.; Porter, A.C.; Tugwell, P.; Moher, D.; Bouter, L.M. Development of AMSTAR: A measurement tool to assess the methodological quality of systematic reviews. BMC Med. Res. Methodol. 2007, 7, 10. [CrossRef] [PubMed] 
10. Shea, B.J.; Reeves, B.C.; Wells, G.; Thuku, M.; Hamel, C.; Moran, J.; Moher, D.; Tugwell, P.; Welch, V.; Kristjansson, E.; et al. AMSTAR 2: A critical appraisal tool for systematic reviews that include randomised or non-randomised studies of healthcare interventions, or both. BMJ 2017, 358, j4008. [CrossRef]

11. Balshem, H.; Helfand, M.; Schünemann, H.J.; Oxman, A.D.; Kunz, R.; Brozek, J.; Vist, G.E.; Falck-Ytter, Y.; Meerpohl, J.; Norris, S.; et al. GRADE guidelines: 3. Rating the quality of evidence. J. Clin. Epidemiol. 2011, 64, 401-406. [CrossRef] [PubMed]

12. Morrison, A.P.; Hunter, J.M.; Halpern, S.H.; Banerjee, A. Effect of intrathecal magnesium in the presence or absence of local anaesthetic with and without lipophilic opioids: A systematic review and meta-analysis. Br. J. Anaesth. 2013, 110, 702-712. [CrossRef] [PubMed]

13. Li, M.; Zhao, X.; Zhang, L.; Niu, X.; Guo, T.; Yang, B.; Liu, Z. Effects and safety of magnesium sulfate on propofol-induced injection pain, a meta-analysis of randomized controlled trials. Int. J. Clin. Exp. Med. 2015, 8, 6813-6821. [PubMed]

14. Zhang, J.; Wang, Y.; Xu, H.; Yang, J. Influence of magnesium sulfate on hemodynamic responses during laparoscopic cholecystectomy: A meta-analysis of randomized controlled studies. Medicine 2018, 97, e12747. [CrossRef] [PubMed]

15. Greenwood, J.; Nygard, B.; Brickey, D. Effectiveness of intravenous magnesium sulfate to attenuate hemodynamic changes in laparoscopic surgery: A systematic review and meta-analysis. JBI Evid. Synth. 2021, 19, 578-603. [CrossRef]

16. Wang, S.C.; Pan, P.T.; Chiu, H.Y.; Huang, C.J. Neuraxial magnesium sulfate improves postoperative analgesia in Cesarean section delivery women: A meta-analysis of randomized controlled trials. Asian J. Anesthesiol. 2017, 55, 56-67. [CrossRef]

17. Ma, S.; Zhang, Y.; Li, Q. Magnesium sulfate reduces postoperative pain in women with cesarean section: A meta-analysis of randomized controlled trials. Pain Pract. Off. J. World Inst. Pain 2021. [CrossRef] [PubMed]

18. Cho, H.K.; Park, I.J.; Yoon, H.Y.; Hwang, S.H. Efficacy of Adjuvant Magnesium for Posttonsillectomy Morbidity in Children: A Meta-analysis. Otolaryngol. Head Neck Surg. Off. J. Am. Acad. Otolaryngol.-Head Neck Surg. 2018, 158, 27-35. [CrossRef] [PubMed]

19. Kawakami, H.; Mihara, T.; Nakamura, N.; Ka, K.; Goto, T. Effect of magnesium added to local anesthetics for caudal anesthesia on postoperative pain in pediatric surgical patients: A systematic review and meta-analysis with Trial Sequential Analysis. PLoS ONE 2018, 13, e0190354. [CrossRef]

20. Xie, M.; Li, X.K.; Peng, Y. Magnesium sulfate for postoperative complications in children undergoing tonsillectomies: A systematic review and meta-analysis. J. Evid.-Based Med. 2017, 10, 16-25. [CrossRef]

21. Lysakowski, C.; Dumont, L.; Czarnetzki, C.; Tramèr, M.R. Magnesium as an adjuvant to postoperative analgesia: A systematic review of randomized trials. Anesth. Analg. 2007, 104, 1532-1539. [CrossRef]

22. Albrecht, E.; Kirkham, K.R.; Liu, S.S.; Brull, R. Peri-operative intravenous administration of magnesium sulphate and postoperative pain: A meta-analysis. Anaesthesia 2013, 68, 79-90. [CrossRef]

23. Chen, C.; Tao, R. The Impact of Magnesium Sulfate on Pain Control after Laparoscopic Cholecystectomy: A Meta-Analysis of Randomized Controlled Studies. Surg. Laparosc. Endosc. Percutaneous Tech. 2018, 28, 349-353. [CrossRef]

24. De Oliveira, G.S., Jr.; Castro-Alves, L.J.; Khan, J.H.; McCarthy, R.J. Perioperative systemic magnesium to minimize postoperative pain: A meta-analysis of randomized controlled trials. Anesthesiology 2013, 119, 178-190. [CrossRef]

25. Guo, B.L.; Lin, Y.; Hu, W.; Zhen, C.X.; Bao-Cheng, Z.; Wu, H.H.; Kaye, A.D.; Duan, J.H.; Qu, Y. Effects of Systemic Magnesium on Post-operative Analgesia: Is the Current Evidence Strong Enough? Pain Physician 2015, 18, 405-418.

26. Murphy, J.D.; Paskaradevan, J.; Eisler, L.L.; Ouanes, J.P.P.; Garcia Tomas, V.A.; Freck, E.A.; Wu, C.L. Analgesic efficacy of continuous intravenous magnesium infusion as an adjuvant to morphine for postoperative analgesia: A systematic review and meta-analysis. Middle East J. Anesthesiol. 2013, 22, 11-20.

27. Ng, K.T.; Yap, J.L.L.; Izham, I.N.; Teoh, W.Y.; Kwok, P.E.; Koh, W.J. The effect of intravenous magnesium on postoperative morphine consumption in noncardiac surgery: A systematic review and meta-analysis with trial sequential analysis. Eur. J. Anaesthesiol. 2020, 37, 212-223. [CrossRef]

28. Li, L.Q.; Fang, M.D.; Wang, C.; Lu, H.L.; Wang, L.X.; Xu, H.Y.; Zhang, H.Z. Comparative evaluation of epidural bupivacaine alone and bupivacaine combined with magnesium sulfate in providing postoperative analgesia: A meta-analysis of randomized controlled trials. BMC Anesthesiol. 2020, 20, 39. [CrossRef]

29. Pascual-Ramírez, J.; Gil-Trujillo, S.; Alcantarilla, C. Intrathecal magnesium as analgesic adjuvant for spinal anesthesia: A meta-analysis of randomized trials. Minerva Anestesiol. 2013, 79, 667-678.

30. Wang, J.; Wang, Z.; Shi, B.; Wang, N. The effect of adding intrathecal magnesium sulphate to bupivacaine-fentanyl spinal anesthesia: A meta-analysis of randomized controlled trials. Medicine 2020, 99, e22524. [CrossRef]

31. Shi, L.; Zhu, H.; Ma, J.; Shi, L.L.; Gao, F.; Sun, W. Intra-articular magnesium to alleviate postoperative pain after arthroscopic knee surgery: A meta-analysis of randomized controlled trials. J. Orthop. Surg. Res. 2021, 16, 111. [CrossRef] [PubMed]

32. Zeng, C.; Li, Y.S.; Wei, J.; Xie, D.X.; Xie, X.; Li, L.J.; Gao, S.G.; Luo, W.; Xiong, Y.L.; Xiao, W.F.; et al. Analgesic effect and safety of single-dose intra-articular magnesium after arthroscopic surgery: A systematic review and meta-analysis. Sci. Rep. 2016, 6, 38024. [CrossRef] [PubMed]

33. Kim, H.Y.; Lee, S.Y.; Lee, H.S.; Jun, B.K.; Choi, J.B.; Kim, J.E. Beneficial Effects of Intravenous Magnesium Administration during Robotic Radical Prostatectomy: A Randomized Controlled Trial. Adv. Ther. 2021, 38, 1701-1712. [CrossRef]

34. Benevides, M.L.; Fialho, D.C.; Linck, D.; Oliveira, A.L.; Ramalho, D.H.V.; Benevides, M.M. Intravenous magnesium sulfate for postoperative analgesia after abdominal hysterectomy under spinal anesthesia: A randomized, double-blind trial. Braz. J. Anesthesiol. 2021, 71, 498-504. [CrossRef] [PubMed] 
35. Guyatt, G.H.; Oxman, A.D.; Vist, G.E.; Kunz, R.; Falck-Ytter, Y.; Alonso-Coello, P.; Schünemann, H.J. GRADE: An emerging consensus on rating quality of evidence and strength of recommendations. BMJ 2008, 336, 924-926. [CrossRef]

36. Beverly, A.; Kaye, A.D.; Ljungqvist, O.; Urman, R.D. Essential Elements of Multimodal Analgesia in Enhanced Recovery after Surgery (ERAS) Guidelines. Anesthesiol. Clin. 2017, 35, e115-e143. [CrossRef]

37. Lyon, F.; Dawson, D. Oucher or CHEOPS for pain assessment in children. Emerg. Med. J. EMJ 2003, 20, 470. [CrossRef]

38. Bijur, P.E.; Silver, W.; Gallagher, E.J. Reliability of the visual analog scale for measurement of acute pain. Acad. Emerg. Med. Off. J. Soc. Acad. Emerg. Med. 2001, 8, 1153-1157. [CrossRef]

39. Bijur, P.E.; Latimer, C.T.; Gallagher, E.J. Validation of a verbally administered numerical rating scale of acute pain for use in the emergency department. Acad. Emerg. Med. Off. J. Soc. Acad. Emerg. Med. 2003, 10, 390-392. [CrossRef]

40. Jakobsen, J.C.; Wetterslev, J.; Winkel, P.; Lange, T.; Gluud, C. Thresholds for statistical and clinical significance in systematic reviews with meta-analytic methods. BMC Med Res. Methodol. 2014, 14, 120. [CrossRef]

41. Higgins, J.P.T.; Thomas, J.; Chandler, J.; Cumpston, M.; Li, T.; Page, M.J.; Welch, V.A. (Eds.) Cochrane Handbook for Systematic Reviews of Interventions; John Wiley \& Sons: Hoboken, NJ, USA, 2021.

42. Castellini, G.; Bruschettini, M.; Gianola, S.; Gluud, C.; Moja, L. Assessing imprecision in Cochrane systematic reviews: A comparison of GRADE and Trial Sequential Analysis. Syst. Rev. 2018, 7, 110. [CrossRef] [PubMed]

43. Kang, H. Trial sequential analysis: Novel approach for meta-analysis. Anesth. Pain Med. 2021, 16, 138-150. [CrossRef] [PubMed] 